\title{
Steady-state mycophenolate mofetil pharmacokinetic parameters enable prediction of systemic lupus erythematosus clinical flares: an observational cohort study
}

Sarah Djabarouti ${ }^{*}$, Dominique Breilh, Pierre Duffau ${ }^{2}$, Estibaliz Lazaro ${ }^{2}$, Carine Greib², Olivier Caubet ${ }^{2}$, Marie-Claude Saux', Jean-Luc Pellegrin², Jean-François Viallard²

\begin{abstract}
Introduction: The aim of this study was to determine whether mycophenolate mofetil (MMF) pharmacokinetics (PK) under combined MMF and prednisone remission-maintenance therapy can predict systemic lupus erythematosus (SLE) clinical flares.

Methods: At inclusion, steady-state PK parameters of the MMF active form, mycophenolic acid (MPA), and its glucuronide metabolite (MPAG) were determined for 25 stable SLE patients without renal manifestations. Disease activity was assessed during 6 months of follow-up. Potential relationships between those entry MMF-PK variables and clinical outcome were analyzed.

Results: MMF controlled disease activity in 17 patients (successes) and failed to do so for 8 others (failures). For failures and successes, respectively, entry MPA areas under the time-concentration curve between 0 and 12 hours $\left(\right.$ AUC $_{0-12 \mathrm{~h}}$ ) (medians: $37.7 \mathrm{vs} 73.1 \mathrm{mg} / \mathrm{h} / \mathrm{L}, P=0.003$ ) and MPA 12-hour trough concentrations $\left(C_{12} \mathrm{~h}\right)$ (medians: 1.5 vs $3.7 \mathrm{mg} / \mathrm{L}, P=0.008$ ) were significantly lower, and inclusion MPAG/MPA $C_{12} \mathrm{~h}$ ratios (medians: 18.7 vs 10.2, $P=0.02$ ) were significantly higher. According to our receiver operating characteristics curve analysis, MPA $C_{12} \mathrm{~h}$ was best able to discriminate a flare during follow-up (93\% sensitivity, $85 \%$ specificity). A 3-mg/L cut-off had 92\% negative-predictive value for developing a flare during follow-up.
\end{abstract}

Conclusions: For our SLE patients without renal manifestations, clinical flares developing under maintenance therapy were associated with steady-state inclusion MPA $C_{12} \mathrm{~h}<3 \mathrm{mg} / \mathrm{L}$.

\section{Introduction}

Systemic lupus erythematosus (SLE) is a chronic remitting-and-relapsing disease that engenders considerable health-care costs for each patient and may require longterm treatment with immunosuppressive agents [1]. Mycophenolate mofetil (MMF), an immunosuppressant widely used to prevent solid organ transplant rejection [2], is being used increasingly to induce SLE remission of minor relapses and to maintain remission after induction therapy [1]. No recommendations for optimal

\footnotetext{
* Correspondence: sarah.djabarouti@chu-bordeaux.fr

'Laboratoire de Pharmacocinétique et Pharmacie Clinique EA2968, Université Victor Segalen Bordeaux 2, Hôpital Haut-Lévêque, CHU de Bordeaux, Avenue de Magellan, 33604 Pessac Cedex, France

Full list of author information is available at the end of the article
}

MMF dose are available for these patients. The dose used to control SLE activity usually ranges from 1.5 to $3 \mathrm{~g}$ daily and is based largely on clinical experience, which is very limited. However, most patients experience periodic disease flares, so maintaining disease control remains a challenge. The lupus flare rate varies among studies, often because of the different populations studied [1].

In the transplant setting, the observed association between drug exposure and the risk of acute rejection has encouraged the routine determination of areas under the time-concentration curve between 0 and 12 hours $\left(\mathrm{AUC}_{0-12 \mathrm{~h}}\right)$ of mycophenolic acid (MPA), the MMF active form [2]. MPA monitoring after MMF 
administration in kidney transplant recipients improved patient outcomes over currently recommended fixeddose strategy [3], although controversy persists [4]. Conversely, for patients with SLE, very few data are available on MMF pharmacokinetic-pharmacodynamic (PK-PD) relationships: an association between disease activity and plasma MPA levels was recently demonstrated in patients with autoimmune diseases [5], as was a concentration-effect relationship between MPA exposure and SLE immunological activity [6].

In light of those findings, we postulated that inadequate immunosuppressive maintenance therapy for SLE reflected insufficient exposure to MPA. This study was conducted to evaluate whether initial steady-state MMF PK parameters in SLE patients without renal manifestations in remission could be predictive of clinical flares. Therefore, we determined those PK parameters for MPA and its main glucuronide metabolite (mycophenolic acid glucuronide, or MPAG) in SLE patients and then examined the parameters' respective predictive values for flares over the next 6 months.

\section{Materials and methods}

\section{Patients}

This single-center, observational study evaluated PK-PD relationships for unselected SLE patients with only extrarenal manifestations, which were defined according to the American College of Rheumatology. Patients were routinely followed between September 2005 and January 2008 in our hospital's internal medicine department. Patients gave written informed consent before being included. The study was approved by our local ethics committee (CHU de Bordeaux).

At the time of inclusion, all patients had completed induction therapy and were on a maintenance regimen comprised exclusively of MMF and low-dose prednisone. The main indications for starting MMF were remission maintenance after induction therapy with pulsed cyclophosphamide or treatment of minor disease relapses of hematological, cutaneous, articular, pulmonary, and/or cardiac SLE manifestations. Five patients who suffered minor relapses had received first-line therapy with hydroxychloroquine or azathioprine and were switched to MMF before inclusion.

Patients who had active renal involvement (proteinuria of greater than $0.5 \mathrm{~g} / 24$ hours with urinary casts or hematuria or both), renal impairment (glomerular filtration rate (GFR) calculated according to the Cockcroft-Gault formula of less than $60 \mathrm{~mL} /$ minute per $1.73 \mathrm{~m}^{2}$ ), and/or hepatic dysfunction were not included, nor were patients who had used cyclosporine, cholestyramine, magnesium- or aluminium-containing antacid, rifampicin, and/or antiviral within the 30 days preceding study entry.
Inclusion steady-state PK parameters were determined for all patients who demonstrated SLE regression or stabilization for at least 1 month. Included patients were those who had been taking a stable MMF dose (1 to $3 \mathrm{~g} /$ day) for at least 1 month with either declining oral prednisone $(0.25$ to $0.5 \mathrm{mg} / \mathrm{kg}$ per day) or prednisone maintenance (5 to $10 \mathrm{mg} /$ day) therapy. MMF dose adaptations were initially made on the basis of tolerance and clinical response, and the inclusion MMF dose was maintained throughout follow-up. To avoid a potential influence of prednisone doses on clinical outcome, a standard predefined tapering regimen was applied to all patients: the prednisone dose had been stable for at least 15 days for all patients before inclusion and was maintained for 2 weeks thereafter and then tapered throughout follow-up (5 mg every 15 days with the objective of withdrawing prednisone for patients receiving 0.25 to $0.5 \mathrm{mg} / \mathrm{kg}$ per day or $1 \mathrm{mg}$ every 15 days for patients under maintenance doses until discontinuation). Patients who discontinued MMF because of intolerance or non-compliance or both were excluded from the final PK-PD analysis.

\section{Pharmacokinetic analysis}

For each patient, nine blood samples were collected in ethylenediaminetetraacetic acid-containing tubes before and $0.5,1,2,3,4,6,8$, and 12 hours after MMF administration. Plasma MPA and MPAG concentrations were determined with chromatographic assays coupled with mass spectrometry [7]. A non-compartmental model with extravascular input for plasma data was used to estimate maximum MPA and MPAG concentrations $\left(\mathrm{C}_{\max }\right)$, times to maximum concentration $\left(\mathrm{T}_{\max }\right)$, 12-hour trough concentrations $\left(C_{12} \mathrm{~h}\right)$, and $\mathrm{AUC}_{0-12 \mathrm{~h}}$, which was estimated with the logarithmic trapezoidal rule, and their MPAG/MPA $\mathrm{AUC}_{0-12 \mathrm{~h}}$ and $\mathrm{C}_{12 \mathrm{~h}}$ ratios were calculated.

\section{Follow-up and clinical outcomes}

The primary outcome measure was the occurrence of clinical flares during the 6 months following entry PK determinations. At each subsequent monthly visit, all patients underwent a complete physical examination and laboratory testing. Serum albumin, aspartate aminotransferase, alanine aminotransferase, and $\gamma$-glutamyltransferase concentrations and GFR were determined, and corticosteroid dose was recorded. Biological analyses were also performed: complete blood cell count, urinalysis, C3 and C4 assays, antinuclear antibody test, and anti-double-stranded DNA.

Clinical outcome was assessed with the Systemic Lupus Erythematosus Disease Activity Index (SLEDAI) score. A flare was defined as an increase in SLEDAI score of at least 3 points since the previous examination 
[8]. Outcomes of patients whose disease remained clinically stable or regressed (SLEDAI score stabilization or improvement) during follow-up were classified as successes, whereas outcomes of patients whose disease was not controlled by the maintenance regimen and who experienced a flare were considered failures.

\section{Statistical analyses}

Patient characteristics and PK data are expressed as median and interquartile range (IQR). Mann-Whitney $U$ test or Fisher exact test (univariate analysis) was used to assess the relationship between each demographic, biological, or PK parameter and clinical outcome. Correlations were established with the Spearman correlation test. Data were analyzed with Statistica software (version 6.1; StatSoft, Créteil, France). Receiver operating characteristic (ROC) curves were analyzed to determine which parameters could best discriminate a clinical flare. Areas under the ROC curves (AUC ROCs) and their 95\% confidence intervals (CIs) were calculated using the method of Hanley and McNeil. AUC ROCs of potentially predictive parameters were compared using Analyse-it software (Analyse-it Software, Ltd., Leeds, UK). The threshold value providing the best trade-off between sensitivity and specificity would be recommended as the discriminant cutoff. A $P$ value of less than 0.05 defined statistical significance.

\section{Results}

\section{Patient characteristics}

The demographic and disease characteristics and inclusion PK parameters of the 26 Caucasian SLE patients without extrarenal manifestations are shown in Table 1. As indicated by the SLEDAI scores, SLE was clinically stable or had regressed for at least 1 month at entry, and all patients met PK steady-state conditions. Twentyone patients ingested $2 \mathrm{~g}$ /day of MMF; daily intake was 1 or $3 \mathrm{~g}$ for 2 patients each, and 1 patient took $1.5 \mathrm{~g} /$ day. Co-medications were oral prednisone (range of 5 to $45 \mathrm{mg} /$ day), calcitriol, and proton-pump inhibitors for all patients. The median MPA $\mathrm{AUC}_{0-12 \mathrm{~h}}$ was $64.7 \mathrm{mg} /$ hour per L, with high between-patient variability, as attested by a coefficient of variation (CV) of $44 \%$.

No significant correlations were observed between MPA AUC ${ }_{0-12 \mathrm{~h}}$ and serum albumin $(r=-0.14, P=0.2)$ or body weight $(r=0.22, P=0.3)$. Plasma MPAG was a mean of 15 -fold higher than MPA (median MPAG $\mathrm{AUC}_{0-12 \mathrm{~h}}$ of $775.3 \mathrm{mg} / \mathrm{hour}$ per L), with wide betweensubject variability of glucuronidation $(\mathrm{CV}=43 \%)$. The MPAG/MPA $\mathrm{AUC}_{0-12 \mathrm{~h}}$ and MPAG/MPA $\mathrm{C}_{12 \mathrm{~h}}$ ratios were highly correlated $(r=0.60, P=0.005)$. A positive correlation was observed between MMF dose and MPA $\mathrm{AUC}_{0-12 \mathrm{~h}}(r=0.58, P=0.01)$ or MPA $C_{12 \mathrm{~h}}(r=0.53$, $P=0.02)$. No significant correlation could be established between the $\mathrm{C}_{12} \mathrm{~h}$ ratios and serum albumin $(r=0.22$, $P=0.3)$, GFR $(r=0.10, P=0.70)$, or prednisone dose $(r=-0.16, P=0.2)$. Lower $C 4$ concentrations tended to be correlated with lower MPA $C_{12} \mathrm{~h}(r=0.44, P=0.08)$.

\section{Relationships between the entry steady-state pharmacokinetic parameters and clinical outcomes}

During follow-up, SLE remained stable or regressed in 17 patients and relapsed in 8. The median MMF duration before the flare was 5 (IQR of 4 to 6) months. The specific SLE manifestations were new arthritis and active glomerulonephritis in 3 patients, arthritis or myositis or both in 2, and vasculitis or cardiorespiratory and neurological symptoms in 1 patient, and cytopenia and myositis in 1 patient. One patient had MMF side effects (diarrhea, nausea, and abdominal pain) requiring its discontinuation and thus was excluded from the PK-PD analysis. The others tolerated it well.

The PK parameters were compared between the 17 successes and 8 failures (Table 1). Patients whose SLE remained clinically stable or regressed during the 6 months of follow-up had baseline MPA $\mathrm{AUC}_{0-12} \mathrm{~h}$ and MPA $C_{12} \mathrm{~h}$ that were significantly higher than those of patients whose disease flared. The latter had significantly higher MPAG/MPA $\mathrm{C}_{12} \mathrm{~h}$ ratios (Figure 1a-c) and significantly lower MPAG $\mathrm{T}_{\max }$. Successes and failures had comparable median inclusion prednisone doses (Table 1). Prednisone doses did not differ statistically between successes and failures at endpoint (respective medians of 5 (IQR of 5 to 5 ) $\mathrm{mg}$ versus 5.5 (IQR of 5.5 to 7.5$) \mathrm{mg} ; P=0.60)$.

\section{Receiver operating characteristic curve analysis}

AUC ROCs were 0.86 (95\% CI 0.71 to 1), 0.89 (95\% CI 0.75 to 0.98 ), and 0.75 (95\% CI 0.56 to 0.90 ), respectively, for MPA $\mathrm{AUC}_{0-12 \mathrm{~h}}$, MPA $\mathrm{C}_{12 \mathrm{~h}}$, and MPAG/ MPA $C_{12}$ h ratio. Comparison of AUC ROCs showed no significant difference between MPA $C_{12} \mathrm{~h}$ and $\mathrm{AUC}_{0-12 \mathrm{~h}}$ $(P=0.77)$. MPA AUC $\mathrm{AU}_{0-12 \mathrm{~h}}$ and $\mathrm{C}_{12} \mathrm{~h}$ were strongly correlated $(r=70, P<0.05)$, but the latter appeared to be better able to discriminate a clinical flare during followup, with $93 \%$ sensitivity and $85 \%$ specificity (Figure 2 ). A threshold of $3 \mathrm{mg} / \mathrm{L}$ had $92 \%$ negative predictive value for developing a flare during follow-up.

\section{Discussion}

Although the majority of our patients received the standard MMF dose of $2 \mathrm{~g} /$ day, broad interpatient CVs for its PK parameters were observed. Moreover, $31 \%$ of our patients relapsed during the 6-month follow-up while on this recommended dose. Considering the attention focused on individualizing MMF therapy for transplantees [3], we postulated that SLE flares occurring under maintenance therapy reflected insufficient exposure to 
Table 1 Inclusion characteristics (of the 26 systemic lupus erythematosus patients) and their ability to predict clinical outcome (univariate analysis)

\begin{tabular}{|c|c|c|c|c|}
\hline Inclusion characteristic & $\begin{array}{c}\text { Total } \\
n=26\end{array}$ & $\begin{array}{c}\text { Successes } \\
n=17\end{array}$ & $\begin{array}{c}\text { Failures } \\
n=8\end{array}$ & $P$ value \\
\hline \multicolumn{5}{|l|}{ Demographic } \\
\hline Females/Males, number & $16 / 10$ & $12 / 5$ & $4 / 4$ & $0.28^{b}$ \\
\hline Age, years & $46(35-61)^{a}$ & $46.5(35-61)$ & $46(38-61)$ & 0.9 \\
\hline Body weight, $\mathrm{kg}$ & $60(56-75)$ & $60(56-75)$ & $62(58-82.5)$ & 0.62 \\
\hline \multicolumn{5}{|l|}{ Disease } \\
\hline Duration before entry, months & $11(7-14)$ & $11(7-12)$ & $10(6-13)$ & 0.30 \\
\hline SLEDAI score & $0(0-2)$ & $0(0-2)$ & $0(0-2)$ & 0.20 \\
\hline $\mathrm{C} 3, \mathrm{~g} / \mathrm{L}$ & $1(0.6-1.1)$ & $1(0.9-1.1)$ & $0.8(0.56-0.96)$ & 0.21 \\
\hline$C 4, g / L$ & $0.2(0.1-0.2)$ & $0.2(0.1-0.2)$ & $0.2(0.16-0.24)$ & 0.78 \\
\hline Anti-double-stranded DNA, IU/mL & $24(6-51)$ & $8(1-32)$ & $38(9-55)$ & 0.19 \\
\hline \multicolumn{5}{|l|}{ Biological } \\
\hline $\mathrm{GFR}, \mathrm{mL} /$ minute & $95(67-125)$ & $95(67-125)$ & $93.5(76-109)$ & 0.64 \\
\hline Albumin, g/L & $40(39.8-43.8)$ & $40(37.9-43.8)$ & $42.8(40-46.9)$ & 0.33 \\
\hline Aspartate aminotransferase, IU/L & $23(19-26)$ & $23(17.5-24.5)$ & $28(20-33)$ & 0.26 \\
\hline Alanine aminotransferase, IU/L & $22(15-29)$ & $19(13-24.5)$ & $25(19-37)$ & 0.21 \\
\hline$\gamma$-Glutamyltransferase, IU/L & $24(17-63)$ & $23(17-21)$ & $42(17-61)$ & 0.92 \\
\hline \multicolumn{5}{|l|}{ Treatment } \\
\hline MMF, g/day & $2(2-2)$ & $2(2-2)$ & $2(2-2)$ & 0.9 \\
\hline Corticosteroids, mg/day & $11(7-35)$ & $10(5-20)$ & $15(7-45)$ & 0.37 \\
\hline Months of MMF therapy & $2(1-3)$ & $2(1-3)$ & $2(1-3)$ & 0.56 \\
\hline Months of corticosteroids & $10(6-13)$ & $10(5-15)$ & $9(5-14)$ & 0.12 \\
\hline \multicolumn{5}{|l|}{ MPA pharmacokinetic parameters } \\
\hline$A \cup C_{0-12 h}, m g / h o u r$ per $L$ & $64.7(38.2-82)$ & $73.1(61.8-95)$ & $37.7(32-43.7)$ & 0.003 \\
\hline $\mathrm{C}_{\max } \mathrm{mg} / \mathrm{L}$ & $16.1(9.5-18.5)$ & $16.3(9.7-17.4)$ & $13.3(7-22.5)$ & 0.69 \\
\hline$T_{\max }$, hours & $1(1-2)$ & $1(1-2)$ & $1.1(1-2)$ & 0.82 \\
\hline$C_{12 h}, \mathrm{mg} / \mathrm{L}$ & $2.4(1.5-4.1)$ & $3.7(2.3-4.9)$ & $1.5(0.6-2.1)$ & 0.008 \\
\hline \multicolumn{5}{|l|}{ MPAG pharmacokinetic parameters } \\
\hline$A \cup C_{0-12 h}, m g / h o u r$ per $L$ & $775.3(475-1,026)$ & $791(635-1,166)$ & $678.8(426-840.6)$ & 0.22 \\
\hline$T_{\max }$, hours & $2(2-3)$ & $2.3(2-3)$ & $1.3(1-2)$ & 0.01 \\
\hline $\mathrm{C}_{12 \mathrm{~h}}, \mathrm{mg} / \mathrm{L}$ & $32.1(24.3-41.9)$ & $34.7(26.4-49.2)$ & $29.8(15.5-40)$ & 0.26 \\
\hline 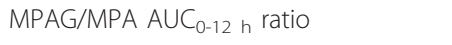 & $11.5(8.3-20.7)$ & $10.9(6.2-14.8)$ & $18.7(14.1-22.7)$ & 0.07 \\
\hline MPAG/MPA $C_{12}$ h ratio & $11.5(6.8-17.3)$ & $10.2(6.3-15)$ & $18.7(11.5-47.2)$ & 0.02 \\
\hline
\end{tabular}

Values are expressed as median (interquartile range) unless stated otherwise. ${ }^{\mathrm{a}}$ Mann-Whitney $U$ test; ${ }^{\mathrm{b}}$ Fisher exact test. AUC $\mathrm{C}_{0-12} \mathrm{~h}$, area under the plasma concentration-versus-time curves for hours 0 to $12 ; C_{12} h$, 12-hour trough concentration; $C_{\text {max }}$ maximal concentration; GFR, glomerular filtration rate (estimated with the Cockcroft-Gault formula); MMF, mycophenolate mofetil; MPA, mycophenolic acid; MPAG, mycophenolic acid glucuronide; SLEDAI, Systemic Lupus Erythematosus Disease Activity Index; $T_{\max }$ time to maximal concentration.

MPA, the MMF active form. Therefore, we examined the possible relationships between MMF PK parameters and SLE activity under maintenance therapy.

Significantly lower MPA AUC $\mathrm{AU}_{0-12 \mathrm{~h}}$ and $\mathrm{C}_{12} \mathrm{~h}$ and higher MPAG/MPA $C_{12} \mathrm{~h}$ ratios were observed at inclusion for patients deemed therapeutic failures during follow-up. Thus, SLE flares were associated with lower plasma MPA concentrations. Our univariate analyses did not find $\mathrm{C} 3$ and $\mathrm{C} 4$ levels or anti-double-stranded DNA antibody positivity to be predictive of such exacerbations. It is commonly accepted that SLE patients may suffer clinical flares without serological changes [9], but in our study, this circumstance could be explained by our small sample size. The only studied parameter differing significantly between clinical successes and failures was entry steady-state MPA exposure.

The ROC curve analysis showed MPA $\mathrm{C}_{12} \mathrm{~h}$ to be as effective a marker as $\mathrm{AUC}_{0-12 \mathrm{~h}}$ to discriminate a clinical flare during follow-up. That observation contradicts findings in the transplantation setting, in which a poor correlation between MPA $C_{12} \mathrm{~h}$ and $\mathrm{AUC}_{0-12 \mathrm{~h}}$ was described [2], making the latter the marker of choice to prevent acute rejection.

According to our results, therapeutic drug monitoring based on the simple measurement of MPA $\mathrm{C}_{12 \mathrm{~h}}$ enabled the prediction of SLE patients' clinical outcomes. 

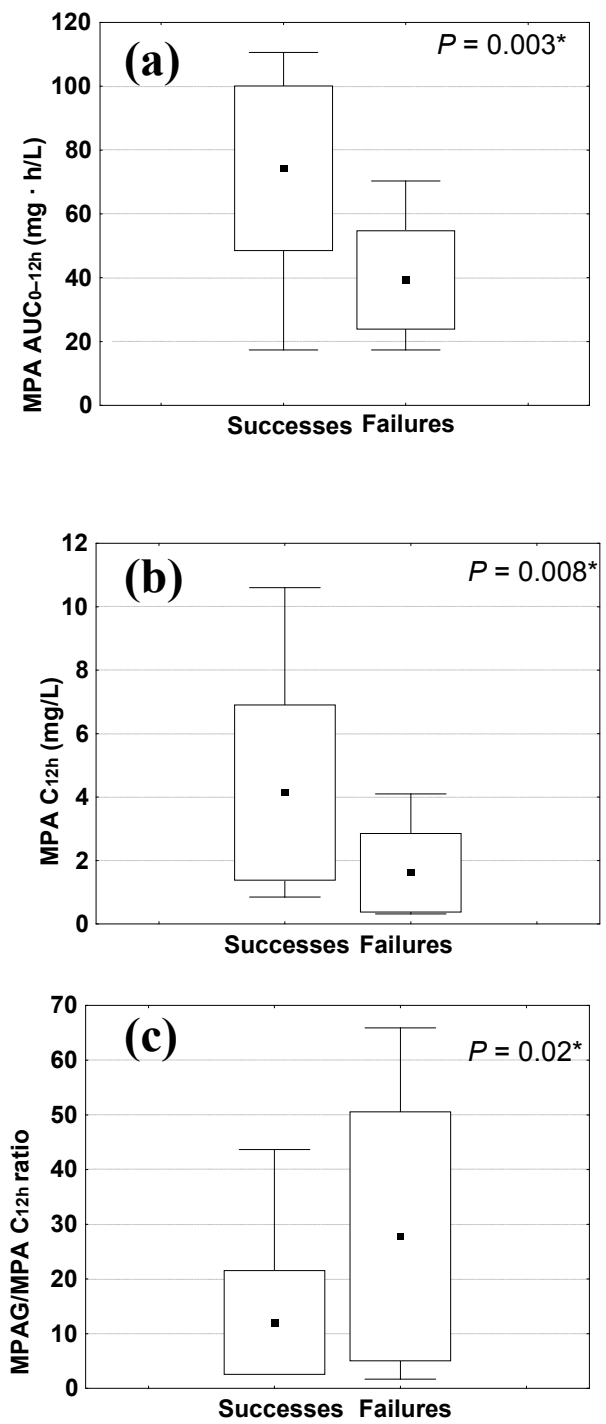

Figure 1 Box plots at baseline for endpoint successes and failures. (a) Area under the plasma concentration-versus-time curves between 0 and 12 hours of mycophenolic acid (MPA AUC 0 -12 h). (b) Twelve-hour trough concentrations of MPA (MPA $C_{12}$ h). (c) Ratios of mycophenolic acid glucuronide (MPAG) to MPA $C_{12}$ h. Black squares inside the boxes are means, the lower and upper box limits are standard deviations, and $\mathrm{T}$ bars correspond to the range. *Mann-Whitney $U$ test.

Notably, our data are in agreement with those of two studies, one conducted on 20 patients with SLE [6] and the other on 39 patients with SLE or vasculitides [5], and confirmed the results reported in the latter. First, our study population was characterized by a mean \pm standard deviation MPA $\mathrm{AUC}_{0-12 \mathrm{~h}}$ of $64 \pm 28 \mathrm{mg} /$ hour per $\mathrm{L}$, which is very close to their value of $66 \pm 22 \mathrm{mg} /$ hour per $\mathrm{L}$ [5]. Those authors also proposed a $\mathrm{C}_{12} \mathrm{~h}$ threshold of $3 \mathrm{mg} / \mathrm{L}$ for MPA monitoring. This threshold provided the best trade-off between sensitivity and specificity, with $92 \%$ negative predictive value in our study.
Unfortunately, our results cannot be compared with those obtained by others, mainly because our inclusion criteria were more restricted, as we enrolled only SLE patients without renal manifestations to ensure clinical homogeneity of the population. Because of the heterogeneous nature of clinical autoimmune disease manifestations, flares are often evaluated using an activity index. More than 20 different lupus activity scales have been used, and the Birmingham vasculitis activity score is used widely in therapeutic studies on systemic vasculitides. We thought that pooling systemic vasculitis and SLE patients would prevent a clear general definition of 'flare' and thereby introduce bias into the determination of efficacy thresholds.

We used one of the best characterized and most routinely used SLE scores and defined a flare as an increase in SLEDAI score of at least 3 points since the previous visit (1 month earlier). Only severe clinical exacerbations requiring therapeutic changes were considered whereas mild and moderate flares were excluded, and this choice might represent a limitation of our study. Another possible limitation is that, owing to the small sample, the relationship between the PK and MMF side effects could not be examined. However, $70 \%$ of our patients had an $\mathrm{AUC}_{0-12} \mathrm{~h}$ of greater than $60 \mathrm{mg} /$ hour per $\mathrm{L}$ without any adverse events. Third, patients were evaluated for only 6 months, and according to Posalki and colleagues [10], although disease activity may regress during the first year of MMF treatment, this immunosuppressant fails to prevent extrarenal flares when continued beyond 2 years. Thus, it is likely that our flare rate would have been higher if follow-up had been longer. Finally, an important limitation is our study's lack of statistical power and the participation of only one center, perhaps explaining why no association was observed between clinical outcome and variables (age, sex, or biological markers) other than PK. Consequently, we were not able to identify confounding variables (for example, age, weight, steroid doses, or sex) in a multivariate analysis [11]. However, for several reasons, we think that prednisone withdrawal could have had only a minor impact on clinical response: low prednisone doses at inclusion (median of $11 \mathrm{mg}$ ), the standard tapering regimen applied to all patients, and comparable median entry and endpoint prednisone doses for successes and failures.

Despite these limitations, our results confirmed the large interpatient CV of the MMF PK parameters for SLE patients, as previously described for transplant recipients [2], showed that therapeutic drug monitoring under MMF steady-state conditions in SLE without renal manifestations predicted the clinical outcome under maintenance therapy, and demonstrated that such PK analyses should be based on MPA $C_{12}$ h, as 


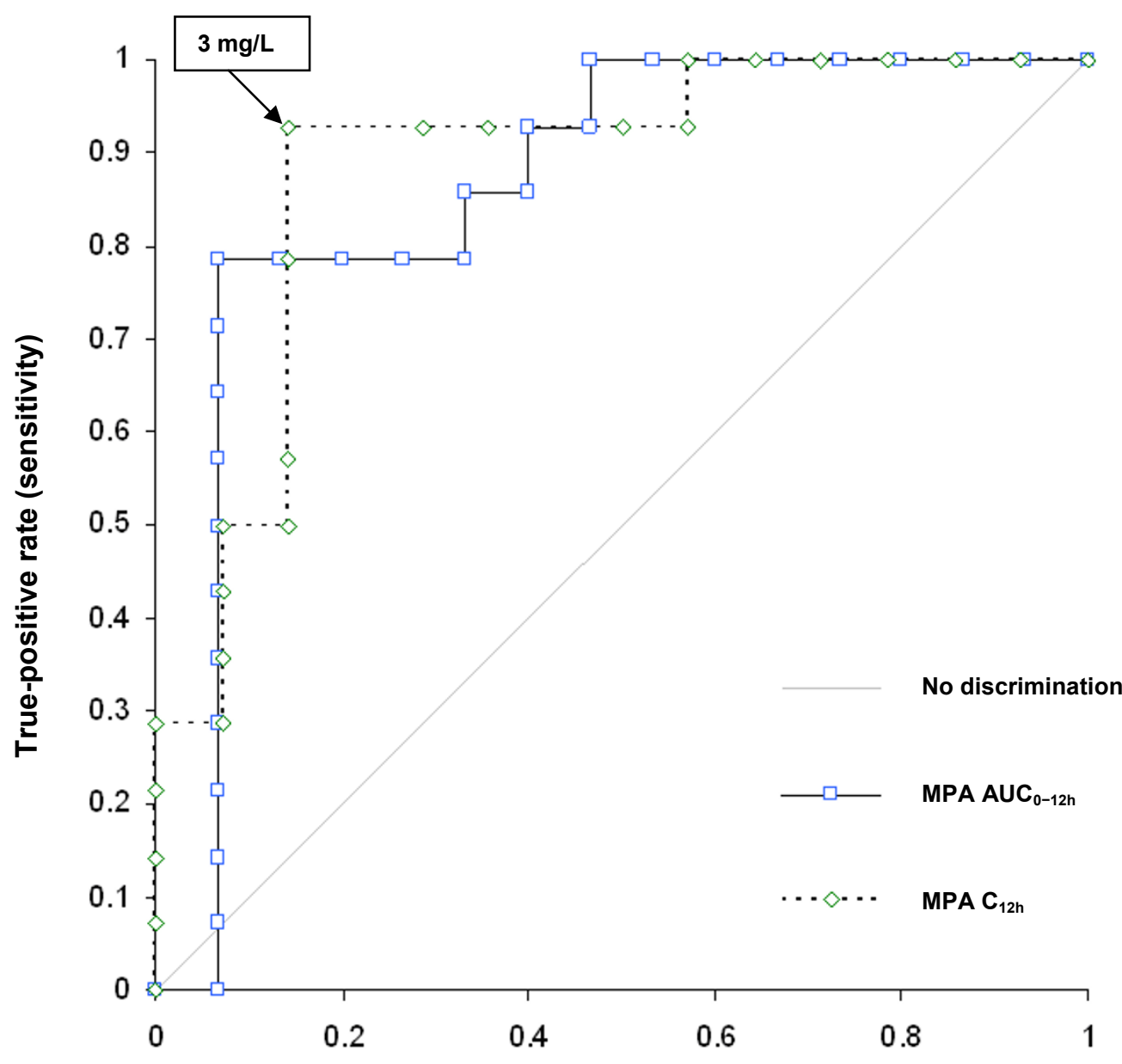

False-positive rate (1 - specificity)

Figure 2 Receiver operating characteristic curve estimates of area under the plasma concentration-versus-time curves between 0 and 12 hours of mycophenolic acid (MPA AUC $0-12 \mathrm{~h}$ ) and 12-hour trough concentration of MPA (MPA $\mathrm{C}_{12 \mathrm{~h}}$ ).

previously reported [5]. We concluded that the discriminant threshold values were highly dependent on the study population and the disease activity index chosen. Indeed, our PK results and proposed efficacy threshold are different from those observed in a cohort that included a majority of patients with lupus nephritis [12]. This discrepancy is logical given the predominant influence of renal function on MPAG and plasma MPA exposure [2].

Therapeutic failures might benefit from optimized dosing. In our opinion, $31 \%$ of our patients should have been prescribed daily MMF doses higher than the standard 2 g. Indeed, the impact of higher MMF doses merits further study. One possible explanation for failures on MMF is the increased clearance suggested by the higher metabolite levels, as the MPAG/MPA $\mathrm{C}_{12 \mathrm{~h}}$ ratio was significantly higher in patients who developed flares. Low serum albumin level, renal dysfunction, and comedications (corticosteroids) are the main factors usually blamed for raising metabolite concentrations [2]. As expected based on our inclusion criteria and sample size, we did not establish any significant correlation between MPAG/MPA $\mathrm{C}_{12}$ h and serum albumin, corticosteroid dose, or GFR. Conversely, the MPAG $\mathrm{T}_{\max }$ was significantly lower for patients failing on MMF, thereby raising a hypothetically increased glucuronidation ability in failures, which favors MPA metabolism and excretion. Glucuronidation might be under genetic control, and this needs to be confirmed in future clinical studies [13]. 


\section{Conclusions}

For our SLE patients without renal manifestations, the occurrence of disease flares under MMF and prednisone maintenance therapy was significantly associated with lower MPA $\mathrm{C}_{12} \mathrm{~h}$. We now recommend a target threshold of $3 \mathrm{mg} / \mathrm{L}$ for our patients. Further studies on larger populations are needed to confirm the ability of MPA PK parameters to predict clinical outcomes of SLE patients.

\section{Abbreviations}

AUC ROC: area under the receiver operating characteristic curve; $A \cup C_{0-12} h$ : area under the time-concentration curve between 0 and 12 hours; $C_{12}$ h: 12hour trough concentration; Cl: confidence interval; CV: coefficient of variation; GFR: glomerular filtration rate; IQR: interquartile range; MMF: mycophenolate mofetil; MPA: mycophenolic acid; MPAG: mycophenolic acid glucuronide; PD: pharmacodynamic; PK: pharmacokinetic; ROC: receiver operating characteristic; SLE: systemic lupus erythematosus; SLEDAI: Systemic Lupus Erythematosus Disease Activity Index; $T_{\max }$ : time to maximum concentration.

\section{Acknowledgements}

We sincerely thank all patients involved in the study, Vianney Jouhet and Sylvie Maurice-Tison for statistical assistance, Janet Jacobson for editorial assistance, and Jean-Marc Bernadou and Vincent Marquais for technical assistance. The authors have no sources of funding to declare.

\section{Author details}

'Laboratoire de Pharmacocinétique et Pharmacie Clinique EA2968, Université Victor Segalen Bordeaux 2, Hôpital Haut-Lévêque, CHU de Bordeaux, Avenue de Magellan, 33604 Pessac Cedex, France. ${ }^{2}$ Service de Médecine Interne et des Maladies Infectieuses, Université Victor Segalen Bordeaux 2, Hôpital Haut-Lévêque, CHU de Bordeaux, Avenue de Magellan, 33604 Pessac Cedex, France.

\section{Authors' contributions}

$\mathrm{SD}, \mathrm{DB}$, and J-FV designed the study, performed experiments, collected data, and wrote the manuscript. PD, EL, CG, and OC followed the patients, collected clinical data, and helped to interpret data. M-CS and J-LP helped to perform experiments, interpret data, and write the manuscript. All authors read and approved the final manuscript.

\section{Competing interests}

The authors declare that they have no competing interests.

Received: 15 June 2010 Revised: 8 November 2010

Accepted: 22 December 2010 Published: 22 December 2010

\section{References}

1. Nannini C, Crowson C, Matteson E, Moder KG: Mycophenolate mofetil is effective in reducing disease flares in systemic lupus erythematosus. Lupus 2009, 18:394-399.

2. Staatz CE, Tett SE: Clinical pharmacokinetics and pharmacodynamics of mycophenolate in solid organ transplant recipients. Clin Pharmacokinet 2007, 46:13-58.

3. Le Meur Y, Büchler M, Thierry A, Caillard S, Villemain F, Lavaud S, Etienne I, Westeel PF, Hurault de Ligny B, Rostaing L, Thervet E, Szelag JC, Rérolle JP, Rousseau A, Touchard G, Marquet P: Individualized mycophenolate mofetil dosing based on drug exposure significantly improves patient outcome after renal transplantation. Am J Transplant 2007, 7:2496-2503.

4. Barraclough KA, Staatz CE, Isbel NM, Johnson DW: Therapeutic monitoring of mycophenolate in transplantation: is it justified? Curr Drug Metab 2009, 10:179-187.

5. Neumann I, Fuhrmann H, Fang IF, Jaeger A, Bayer P, Kovarik J: Association between mycophenolic acid 12-h trough levels and clinical endpoints in patients with autoimmune diseases on mycophenolate mofetil. Nephrol Dial Transplant 2008, 23:3514-3520.
6. Roland M, Barbet C, Paintaud G, Magdelaine-Beuzelin C, Diot E, Halimi JM, Lebranchu $Y$, Nivet $H$, Büchler M: Mycophenolate mofetil in patients with systemic lupus erythematosus: a prospective pharmacokinetic study. Lupus 2009, 18:441-447.

7. Djabarouti S, Duffau P, Xuereb F, Lazaro E, Greib C, Gordien JB, Caubet O, Saux MC, Viallard JF, Pellegrin JL, Breilh D: Measurement of total and unbound mycophenolic acid and its glucuronide by liquid chromatography coupled with mass spectrometry. Application to pharmacokinetic study of mycophenolate mofetil in patients with autoimmune diseases. Chromatographia 2009, 70:939-945.

8. Petri M, Genovese M, Engle E, Hochberg M: Definition, incidence, and clinical description of flare in systemic lupus erythematosus. A prospective cohort study. Arthritis Rheum 1991, 34:937-344.

9. Walz LeBlanc BA, Gladman DD, Urowitz MB: Serologically active clinically quiescent systemic lupus erythematosus-predictors of clinical flares. J Rheumatol 1994, 21:2239-2241.

10. Posalski J, Ishimori M, Wallace DJ, Weisman MH: Does mycophenolate mofetil prevent extra-renal flares in systemic lupus erythematosus? Results from an observational study of patients in a single practice treated for up to 5 years. Lupus 2009, 18:516-521.

11. Morissette P, Albert C, Busque S, St-Louis G, Vinet B: In vivo higher glucuronidation of mycophenolic acid in male than in female recipients of a cadaveric kidney allograft and under immunosuppressive therapy with mycophenolate mofetil. Ther Drug Monit 2001, 23:520-525.

12. Zahr N, Arnaud L, Marquet P, Haroche J, Costedoat-Chalumeau N, Hulot JS, Funck-Brentano C, Piette JC, Amoura Z: Mycophenolic acid area under the curve correlates with disease activity in lupus patients treated with mycophenolate mofetil. Arthritis Rheum 2010, 62:2047-2054.

13. Bernard O, Guillemette C: The main role of UGT1A9 in the hepatic metabolism of mycophenolic acid and the effects of naturally occurring variants. Drug Metab Dispos 2004, 32:775-778.

\section{doi:10.1186/ar3202}

Cite this article as: Djabarouti et al: Steady-state mycophenolate mofetil pharmacokinetic parameters enable prediction of systemic lupus erythematosus clinical flares: an observational cohort study. Arthritis Research \& Therapy 2010 12:R217.

\section{Submit your next manuscript to BioMed Central and take full advantage of:}

- Convenient online submission

- Thorough peer review

- No space constraints or color figure charges

- Immediate publication on acceptance

- Inclusion in PubMed, CAS, Scopus and Google Scholar

- Research which is freely available for redistribution

Submit your manuscript at www.biomedcentral.com/submit
C Biomed Central 